\title{
Freeze-out from HBT and Coulomb Effects
}

\author{
H. Heiselberg ${ }^{\mathrm{a}}$
}

aNORDITA,

Blegdamsvej 17, DK-2100 Copenhagen O., Denmark

The freeze-out of hot and dense hadronic matter formed in relativistic nuclear collisions is probed by HBT interferometry of identical pions, kaons, etc. Coulomb repulsion/attraction of positive/negative particles show up at small particle momenta and is also very sensitive to the freeze-out conditions. The source sizes and times freeze-out are extracted from $\pi^{-} / \pi^{+}$spectra and HBT radii and compared.

\section{Coulomb Effects in Single Particle Spectra}

An asymmetry in the number of opposite charge pions has been observed at intermediate energies and has recently also been identified in heavy ion collisions at energies around 1 $A \cdot \mathrm{GeV}$ [1], $11.4 A \cdot \mathrm{GeV}$ [2], and $158 A \cdot \mathrm{GeV}$ [3]. The ratio of negative to positive pions at low pion momenta is $\sim 3$ at SIS energies, but only $\sim 1.6$ at AGS and SPS energies for the central collisions of heavy nuclei as $A u$ or $P b$. This effect can be explained by the Coulomb interaction between the produced pions and the positive charge from the reaction partners.

At ultrarelativistic collision energies it is important to include the rapid expansion of the system and its net charge as well as retardation effects when considering the Coulomb effect on particles. For rapid longitudinal Bjorken expansion we find (see [4] for details and more elaborate calculations) that the Coulomb field decrease with time as $\sim t^{-1}$ and is proportional to the net charge (proton) rapidity distribution $d N^{c h} / d y$. The pions are assumed to freeze-out at time $\tau_{f}$ from a cylindrical symmetric source of transverse size $R_{f}$. On average a charged pion of transverse momentum $p_{\perp, 0}$ at freeze-out and final momentum $p_{\perp}$ receives a momentum change or "Coulomb kick" $\pm p_{c}$ for $\pi^{ \pm}$respectively

$$
p_{c} \equiv\left|\mathbf{p}_{\perp}-\mathbf{p}_{\perp, 0}\right| \simeq 2 e^{2} \frac{d N^{c h}}{d y} \frac{1}{R_{f}} .
$$

The momentum change is only a slowly decreasing function of the freeze-out time. For example, $p_{c}$ varies $\pm 10 \%$ for $p_{\perp} \tau_{f} / m_{\perp} R_{f}$ in the range $0.5-0.8$.

The Coulomb effect on the transverse particle distribution functions can be derived from particle conservation $d N / d^{2} p_{\perp}=\left(p_{\perp, 0} / p_{\perp}\right) d N_{0} / d^{2} p_{\perp, 0}$. The pion spectra are well reproduced by $d N^{0} / d^{2} p_{\perp, 0} \propto \exp \left(-m_{\perp, 0} / T\right)$ with $T \simeq 150 \mathrm{MeV}$ at both AGS and SPS energies. Defining $m_{\perp}^{ \pm}=\sqrt{m^{2}+\left(p_{\perp} \pm p_{c}\right)^{2}}$ the ratio is

$$
\frac{\pi^{-}}{\pi^{+}} \equiv \frac{d N^{-} / d^{2} p_{\perp}}{d N^{+} / d^{2} p_{\perp}}=\left\langle\frac{\pi^{-}}{\pi^{+}}\right\rangle \frac{p_{\perp}+p_{c}}{p_{\perp}-p_{c}} \exp \left(\frac{m_{\perp}^{-}-m_{\perp}^{+}}{T}\right) .
$$


The pion ratio predicted by (2) is compared to AGS and SPS data in Fig. 1. We use the experimental values for the total pion ratio $\left\langle\pi^{-} / \pi^{+}\right\rangle \simeq 1.27$ and 1.05 at AGS and SPS energies, respectively. The experimental data is well reproduced with $p_{c}^{A G S} \simeq 20 \mathrm{MeV} / \mathrm{c}$ and $p_{c}^{S P S} \simeq 10 \mathrm{MeV} / \mathrm{c}$. From the measured proton rapidity distribution, $d N_{A G S}^{p} / d y \simeq 70$ and $d N_{S P S}^{p} / d y \simeq 37$, and Eq.(1) we can now extract the size of the systems at freeze-out: $R_{f} \simeq 10 \mathrm{fm}$, at both AGS and SPS.

The Coulomb effect is stronger at lower energies due to slow expansion of the net charge. A spherical and static charge of size $R$ changes the particle energies by the

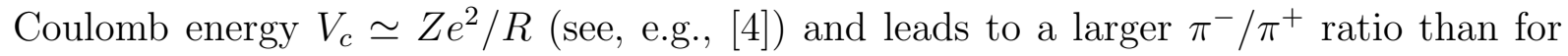
the rapidly expanding case Eq. (11) as seen in Fig. (1). With $\left\langle\pi^{-} / \pi^{+}\right\rangle \simeq 1.9$ we extract from the data $V_{c} \simeq 27 \mathrm{MeV}$. With $Z=110$ for the $A u+A u$ collisions with $14 \%$ centrality at SIS we obtain $R \simeq 8 \mathrm{fm}$.

The smaller charge in $S+S$ collision $\left(d N^{p} / d y \simeq 4\right.$ at SPS) leads to a smaller $\pi^{-} / \pi^{+}$ ratio that is compatible with data [3, 4 .

Kaons are heavier than pions and are less affected by Coulomb fields. Yet a small enhancement in the $K^{-} / K^{+}$ratio at low $m_{\perp}$ is predicted [4]. Preliminary data from NA44 [3] shows a flat $K^{-} / K^{+}$ratio indicating that other physics is needed such as strong interactions counter-balancing the Coulomb fields.

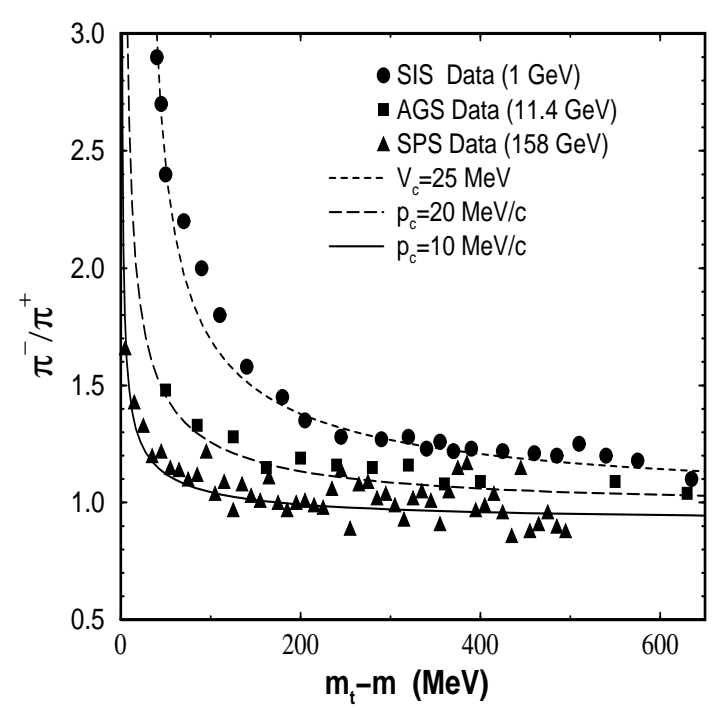

Figure 1. $\pi^{-} / \pi^{+}$ratios vs. $m_{\perp}$ at SIS, AGS and SPS energies. Curves show model results (see text).

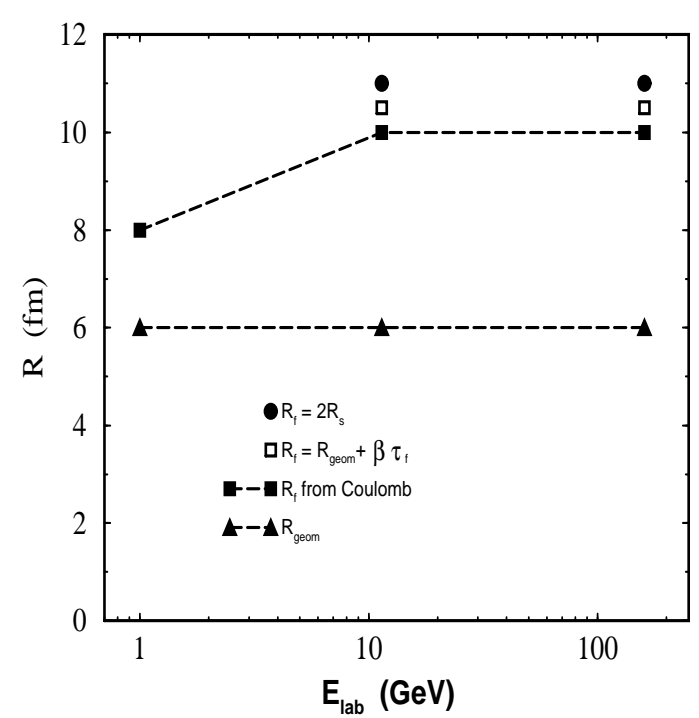

Figure 2. Freeze-out radii extracted from Coulomb effects and HBT (see text) are larger than geometrical radii. 


\section{HBT Radii}

Bose-Einstein correlations (HBT) in relativistic heavy ion collisions depend sensitively on the freeze-out condition. The source sizes are measured in in the three directions, $R_{s}, R_{o}, R_{l}$, referred to as the sideward, outward and longitudinal HBT radii. The theoretical interpretation is, however, very model dependent.

For a transparent source the outward HBT radius is larger than the sideward [5,6]

$$
R_{o}^{2}=R_{s}^{2}+\beta_{o}^{2} \sigma(\tau)
$$

when opacity effects and transverse flow is ignored. Here, $\sigma(\tau)$ is the temporal fluctuations and contains the summed squares of the duration of emission, the average of short lived resonance life-times and fluctuations in these [7]. The difference between $R_{o}$ and $R_{s}$ is due to the temporal extent of the source during which the particles with outward velocity $\beta_{o}=p_{\perp} / m_{\perp}$ travel on average a distance $\beta_{o} \sigma(\tau)^{1 / 2}$ towards the detector.

In opaque sources and hydrodynamic models particles are only emitted from the surface contrary to the transparent sources where particle freeze-out in volume. By expanding for small relative momenta, $q_{i} \ll \hbar / R_{i}$, one can express the HBT radii in terms of various fluctuations. For very opaque sources of transverse size $R$, where the particle mean free path is short $\lambda_{m f p} \ll R$, the HBT radii can be evaluated [8] ( $R$ is twice the commonly used gaussian radius parameter)

$$
\begin{aligned}
R_{s}^{2} & =\frac{1}{3} R^{2}-\frac{1}{6} \lambda_{m f p}^{2} \\
R_{o}^{2} & =\left(\frac{2}{3}-\left(\frac{\pi}{4}\right)^{2}\right) R^{2}+\beta_{o}^{2} \sigma(\tau)+\left(\frac{7}{6}-\frac{\pi^{2}}{32}\right) \lambda_{m f p}^{2} .
\end{aligned}
$$

In 8$]$ the HBT radii are also calculated with transverse flow and moving surfaces. Notice the different result for the transparent sources, for which $R_{s}=R / 2$ and $R_{o}$ is given by (3). The opaque source has a much smaller transverse spatial extent in the outward as in the sideward direction, $\left(2 / 3-(\pi / 4)^{2}\right) \simeq 0.05 \ll 1 / 3$, because the emission now take place in a narrow surface layer of order the mean free path.

In relativistic heavy ion collisions the outward and sideward HBT radii are measured to be similar [3,9, 10] and in a few cases the outward is even measured to be smaller than the sideward HBT radius [3,9] contradicting Eq. (3). According to Eq. (3) this implies that particles freeze-out suddenly, $\sigma(\tau)^{1 / 2} \ll R_{i}$, as in a "flash", in particular when resonance life-times are included. However, both the opacity effect and transverse flow reduce $R_{o}$ more than $R_{s}$ and so it is possible that $R_{o}<R_{s}$. Present experimental results do not allow us to draw conclusions from the small difference between $R_{o}$ and $R_{s}$ since error bars are of the same size. However, one cannot use Eq. (3) to extract the duration of emission since opacity and transverse flow can have significant effects. Wiedemann et al. [6] have analysed the NA49 data and exclude opacity effects. However, in the NA49 data $R_{o}>R_{s}$ [10]. It would therefore be interesting to investigate whether opacity effects are needed to describe the NA44 data for which $R_{o} \lesssim R_{s}$.

\section{Summary}

The $\pi^{-} / \pi^{+}$ratios at SIS, AGS and SPS energies can be explained by Coulomb effects with freeze-out radii about twice the geometrical radii of the colliding nuclei. Similar 
freeze-out radii can be obtained from HBT analyses of $R_{s}$ and $R_{l}$. The following freezeout radii were extracted (see Fig. 2):

i) From $\pi^{-} / \pi^{+}$ratio we obtained best fits for the Coulomb kick $p_{c} \simeq 2 e^{2}\left(d N^{p} / d y\right) / R_{f}$ at AGS and SPS and $V_{c}=Z e^{2} / R$ at SIS energies. These values allowed us to estimate the freeze-out radii.

ii) For a source of constant pion density the sharp cut-of radius is given by $R_{f}=2 R_{s}$, where the sideward HBT radius $R_{s} \simeq 5-6 \mathrm{fm}$ in central $A u$ or $\mathrm{Pb}$ collisions at AGS [12] and SPS 9, 10.

iii) In a longitudinally expanding system with Bjorken scaling the freeze-out time is

related to the longitudinal HBT radius by $\tau_{f}=R_{l} \sqrt{m_{\perp} / T}$. From the AGS and SPS data $R_{l} \simeq 5.5 \mathrm{fm}$ [12,9] we estimate $\tau_{f} \simeq 8 \mathrm{fm} / \mathrm{c}$. Assuming an initial geometrical radius $R_{\text {geom }} \simeq 6 \mathrm{fm}$ (for $\sim 15 \%$ centrality) with transverse flow $\beta \simeq 0.5-0.6 c$ [11], we find a radius of $R_{f}=R_{\text {geom }}+\beta \tau_{f} \simeq 11-12 \mathrm{fm}$.

iv) At present it is difficult to extract information on freeze-out from $R_{o}$ since it is very model dependent. Opacity and transverse flow effects reduce it whereas duration of emission and resonances increase it with respect to $R_{s}$.

We conclude that both freeze-out times extracted from the longitudinal and transverse HBT radii are at AGS and SPS energies and within experimental uncertainty compatible with those found from $\pi^{-} / \pi^{+}$ratios as seen from Fig. 2. The freeze-out radii are substantially larger than the geometrical radii $R_{\text {geom }} \simeq 6 \mathrm{fm}$ for near central collisions $A u$ or $\mathrm{Pb}$ nuclei indicating that expansion takes place before freeze-out.

Acknowledgements to my collaborators Barz, Bondorf, Gårdhøje and Vischer.

\section{REFERENCES}

1. A. Wagner et al., preprint Th Darmstadt, IKDA 95/9 (1995) and submitted to Phys. Lett.; D. Pelte et al., Z.Phys.A357 (1997) 215

2. L. Ahle et al., Nucl. Phys. A610 (1996) 139c

3. H. Bøggild et al. (NA44), Phys. Lett. B 372, 343 (1996)

4. H.W. Barz, J. Bondorf, J.J. Gårdhøje, and H. Heiselberg, Phys. Rev. C56 (1997) 1553. nucl-th/9711064

5. T. Csörgö and B. Lörstad, Phys. Rev. C54 (1996) 1390; Z. Physik C71, 491 (1996);

6. S. Chapman, J.R. Nix, and U. Heinz, Phys. Rev. C52, 2694 (1995); U.A. Wiedemann et al., these proceedings.

7. H. Heiselberg, Phys. Lett. B379 (1996) 27.

8. H. Heiselberg and A.P. Vischer, Z. Phys. C in press (nucl-th/9609022); Phys. Lett. B 1998, in press (nucl-th/9703030).

9. A. Franz, NA44, Nucl. Phys. A 610 (1996) 240c; and these proc.

10. NA49 coll., K. Kadija et al., Nucl. Phys. A 610 (1996) 248c; S.V. Afanasev et al., Nucl. Phys. A610, 76c (1996); and these proc.

11. I.G. Bearden et al. (NA44), Phys. Rev. Lett. 78, 2080 (1997).

12. E877 coll., J. Barrette et al., Nucl. Phys. A610 (1996) 227c; and these proc. 